\title{
GESTIÓN DE OPERACIONES Y TALENTO HUMANO: UN MODELO DE ELECCIÓN DISCRETA.
}

\section{MANAGEMENT OF OPERATIONS AND HUMAN TALENT: A MODEL OF DISCRETE ELECTION. \\ GESTÃO DE OPERAÇÕES E TALENTO HUMANO: UM MODELO DE ELEIÇÃO DISCRETA.}

\section{Por: RODRIGUEZ LUNA_Raúl Enrique, ROSENSTIEHL MARTINEZ_José Luis}

Candidato a doctor en Economía de la Universidad de Zulia, Venezuela. Magister en Economía Universidad Nacional de Colombia. Profesor Investigador Universidad Cooperativa de Colombia, Santa Marta. Email: raul.rodriguez@campusucc.edu.co

Candidato a doctor en Ciencias Gerenciales y Magister en Gerencia de Mercadeo de la Universidad Rafael Belloso Chacin, Venezuela. Investigador Universidad Cooperativa de Colombia, Santa Marta. Email: jose.rosenstieh1@campusucc.edu.co

Recibido: 30 de mayo de 2018 Aprobación definitiva: 6 de diciembre de 2018

\section{DOI: http://dx.doi.org/10.22267/rtend.181902.99}

\section{RESUMEN}

El objetivo de este trabajo es determinar si existe alguna probabilidad de que el bajo desempeño en la gestión de operaciones logística (GO) en América Latina (AL) se esté presentando por la falta de talento humano especializado en actividades logística (TH). El instrumento de recolección de información se aplicó a una muestra de 126 empresas de menor tamaño dedicada a realizar operaciones logísticas en esta región, el instrumento utilizado como recurso fue la red de Internet; los datos se analizaron mediante análisis factorial confirmatorio y Regresión Logística, utilizando los programas estadísticos SPSS v.23 Por otra parte, se notan varios asuntos, la importancia de la gestión de operaciones (OG) 
y el talento humano $(\mathrm{TH})$ aunque estas, se encuentran relacionadas, a nivel investigativo es escasa la evidencia que estudia su relación para (AL), el trabajo encuentra evidencia en favor de la hipótesis que predice, el (TH) influye en el rendimiento de las $(\mathrm{OG})$, esta, la hipótesis parte de la teoría de la eficiencia técnica a partir de los trabajos de Farrel (1957).

Palabras Clave: Gestión de operaciones, talento humano, logística, modelos de elección discreta, eficiencia técnica.

JEL: M11, C25, C01

\section{ABSTRACT}

The objective of this paper is to determine if there is any likelihood that the low performance in logistics operations management (GO) in Latin America (LA) is being presented due to the lack of human talent specialized in logistics activities (TH). The information collection instrument was applied to a sample of 126 smaller companies dedicated to carry out logistics operations in this region, the instrument used as a resource was the Internet network; the data were analyzed by confirmatory factor analysis and Logistic Regression, using the statistical programs SPSS v.23 On the other hand, several issues are noted, the importance of operations management $(\mathrm{OG})$ and human talent $(\mathrm{TH})$ even though these are find related, at the research level is little evidence that studies their relationship for (AL), the work finds evidence in favor of the hypothesis that predicts, the (TH) influences the performance of (OG), this, the hypothesis part of the theory of technical efficiency from the works of Farrel (1957).

Keywords: Operations management, human talent, logistics, discrete choice models, technical efficiency.

JEL: M11, C25, C01

\section{RESUMO}

O objetivo deste trabalho é determinar se existe alguma probabilidade de que o baixo desempenho na gestão de operações logísticas (GO) na América Latina (LA) esteja sendo apresentado devido à falta de talentos humanos especializados em atividades de logística (TH). O instrumento de coleta de informações foi aplicado a uma amostra de 126 empresas menores dedicadas a realizar operações logísticas nessa região, o instrumento utilizado como recurso foi a rede Internet; 
os dados foram analisados por análise fatorial confirmatória e Regressão Logística, utilizando-se os programas estatísticos SPSS v.23 Por outro lado, várias questões são apontadas, a importância da gestão das operações (OG) e do talento humano $(\mathrm{TH})$ encontrar relacionado, ao nível de pesquisa é pouca evidência de que estuda sua relação para (AL), o trabalho encontra evidências em favor da hipótese que prediz, o (TH) influencia o desempenho de (OG), esta, a parte da hipótese da teoria da eficiência técnica a partir dos trabalhos de Farrel (1957).

Palavras-chave: Gestão de operações, talento humano, logística, modelos de escolha discreta, eficiência técnica.

JEL: M11, C25, C01

\section{INTRODUCCIÓN.}

Este trabajo aporta al tema de la innovación en tecnología a partir de los trabajos formales de eficiencia técnica, que inician formalmente a principios de los cincuenta, con los trabajos de Koopmans (1951), Debreu (1951) y Farell (1957). Aunque recientemente los empresarios ven en la planificación de innovación tecnológica el tema de la eficiencia técnica (ET) e intentan incorporar estrategias relacionadas con la gestión de operaciones (GO) y ven a esta como sinónimo de rentabilidad, reducción de inventarios, aunque han incorporado el concepto de gestión de materiales para alcanzar el óptimo del servicio a partir de la minimización de costos, recurren muchas veces a mejorar los niveles de productividad a partir de estrategias logística como un modelo estratégico de eficiencia. Este trabajo va en esa dirección, intentando evaluar si la falta de talento humano especializado en actividades logísticas probablemente afecta el desempeño de la (GO) lo que conllevaría probablemente a la falta de innovación en el diseño de productos y procesos en las empresas de menor tamaño en América Latina (AL).

Sin embargo, una de las dificultades en América Latina (AL), está relacionado con la (ET) y específicamente en asuntos relacionados con bajos niveles de desempeño logístico que tienen que ver principalmente con una inadecuada infraestructura, falta de inversión, bajos desempeño de actores involucrados en la cadena de abastecimiento, restricciones relacionadas con legislación de cada país, bajos niveles de acumulación de capital humano en sentido estricto, 
muchos trabajadores que al no ser especialistas en actividades logísticas no contribuyen con la optimización de los costos de operación, o con la gestión de operaciones, ni mucho menos con el servicio a sus clientes (Kirby \& Brosa, 2011).Esta situación, junto con otros factores como los costos logísticos como porcentaje del PIB evidencia que estos, los costos logísticos en América Latina, son altos en comparación con Singapur y países del OCDE Kogan \& Guash (2006). En este sentido, se propuso responder la siguiente pregunta: ¿existiría alguna probabilidad de que el bajo desempeño en la (GO) en (AL) se estaría presentando por la falta de $(\mathrm{TH})$ especializado en actividades logística en esta región?

\section{REVISIÓN DE LA LITERATURA.}

En cuanto a los aspectos conceptuales, es pertinente tomar en cuenta lo expresado por, Langley (1986); Carrasco (2000); García (2004); Trujillo (2005); Christopher (1994); Lambert et al (1998); Ballou (1999); Stock et al (2000); Sarache et al. (S/A). A comienzo de los noventa, se comenta sobre el tema de la logística, hoy logística pasa a formar parte integral de la cadena de suministros, intentando integrar los procesos operativos y de capital humano.

En este sentido, (Cohen S. y Roussel J., 2005), Chopra y Meindl (2008) y Bowersox et al (1986) consideran que es la planeación, el abastecimiento y la producción, los factores determinantes de la cadena de suministro incluye no solamente al fabricante y al proveedor, sino también a los transportistas, almacenistas, vendedores al detalle e incluso a los mismos clientes. Según la literatura económica, el desempeño en las compras es considerado un importante elemento del desempeño corporativo, sin embargo, la medición de este desempeño y su comparación con otras áreas de compras y transporte, ha probado ser muy difícil. Desde la perspectiva de Harland et al (1999) los costos de producción, la calidad y la velocidad de respuesta al cliente, así como los costos de transporte, calidad y los tiempos de entrega; en esta misma ruta, Rojas (2014) establece que compras, integración de proveedores, las asociaciones de comprador-proveedor, la fuente de gestión de bases, alianzas estratégicas de proveedores, la sincronización de la cadena de suministro y, por último, simplemente Supply Chain Management son los factores determinantes de la gestión de operaciones, al respecto, Stock \& Lambert (2000) señalan que es 
la integración organizacional, proveedores, minoristas y clientes, las estrategias corporativas, la red de la cadena de abastecimiento, incluyendo al cliente final seria los factores determinantes de la gestión de operaciones. Para (Simichi, et al, 2000) y Jiménez (2000 son los detallistas los quienes deben entregar las cantidades correctas, en los lugares correctos y en el tiempo preciso, buscando el menor costo, Beamon (1998), considera que los proveedores, fabricantes, distribuidores y minoristas, quienes determinan el desempeño de la gestión.

Al respecto, Servera, Gil y Fuentes (2009), señalan que en la práctica empresarial y en el estudio académico de la logística, la incorporación de los conceptos "calidad" primero y "valor" después, Es a mediados de los años 90, cuando el estudio de la logística empieza a centrarse en su capacidad para entregar calidad y generar valor (Mentzer et al 2004), y hoy, en palabras de Ballou (2004:13), se acepta que "la logística gira en torno a la creación de valor: valor para los clientes, los proveedores y los accionistas de la empresa".

Sin embargo, en la actualidad no existe suficiente claridad acerca de los factores que determinan la probabilidad de ocurrencia del bajo desempeño de la gestión de operaciones logística por la falta de talento humano especializado en algunas empresas de menor tamaño en América Latina, Arciniegas (1998), Ariza (1997), Carrasco (2000) y Lambert et al (1998); no obstante, muchos estudios han encontrado que la (GO) está determinada por factores de gestión del conocimiento de orientación al cliente, capacidad del empleado y capacitación relacionada con el trabajo tuvieron una influencia variable en las dimensiones de calidad y eficiencia del rendimiento del sistema de servicio. Jayaram y $\mathrm{Xu}$, (2016).

De hecho, Henry, Patuwo y Hu, (1998) en un trabajo reciente respecto a "The human factor in advanced manufacturing technology adoption: An empirical analysis", examinan los factores de éxito críticos para la adopción e implementación de tecnología de fabricación avanzada.evalúan empíricamente la hipótesis de que las variables de gestión más asociadas con el factor humano en los proyectos de automatización por sí solas pueden diferenciar a las empresas que tienen éxito en la adopción de las tecnologías de aquellas que no tienen tanto éxito. Al respecto, Neumann, \& Dul (2010) plantea que, son escasos los aportes empíricos que abordan la articulación entre la gestión humana y estrategia de 
operaciones, sin embargo, Ahmad \& Schroeder (2003). En The impact of human resource management practices on operational performance: recognizing country and industry differences. Journal of Operations Management, evaluaron en cuatro países el impacto que siete prácticas de gestión humana (seguridad en el empleo, contratación selectiva, uso de equipos de trabajo y descentralización, compensación asociada al desempeño, entrenamiento extensivo, diferencia de status e información compartida) tenían sobre el desempeño en las operaciones, medida globalmente a través del desempeño en seis prioridades con respecto a los competidores del mercado: costo unitario, calidad, entregas, flexibilidad y velocidad en la introducción de nuevos productos.

\section{METODOLOGÍA.}

\section{TIPO DE INVESTIGACION.}

Este trabajo es de tipo exploratorio con un enfoque cuantitativo y un alcance correlacional. Los datos provienen de algunas empresas del sector logístico en AL, que colaboraron con el estudio, para la toma de los datos se utilizaron empresas de menor tamaño por número de trabajadores del sector logístico en AL de acuerdo con la ficha técnica de la Tabla 1 y cuyas actividades logísticas, son homologas a CIIU 5229 (Revisión 4 adaptada para Colombia CIIU Rev. 4 A.C.) para las economías objetos de estudio.

En relación con el tamaño de la muestra para la realización del modelo logístico se utilizó el criterio Freeman (1987) el cual afirma "el tamaño de muestra ha de ser unas diez veces el número de variables independientes a estimar más uno," p.50. formalmente $[\mathrm{n}=10 *(\mathrm{k}+1)]$ donde, $\mathrm{n}$ representa tamaño de la muestra $\mathrm{k}$, es el número de parámetros a estimar para efectos de este trabajo es uno (1). En este sentido un principio esencial en la determinación del tamaño de una muestra es que la aproximación utilizada se corresponda con los objetivos y el diseño de la investigación y con el tipo de análisis que se está planeando.

Para la selección de las variables de estudio, se tuvieron en cuenta los trabajos de Jayaram y Xu, (2016); Jayaram y Vickery (1999); Da Silveira \& Sousa (2010), Furlan. \& Dal (2011); Goodridge (1986); Patrick y Dul, (2010) ), quienes analizaron variables relacionadas con los determinantes de la calidad y el desempeño de la eficiencia en las operaciones de servicio, factores de gestión del 
conocimiento de orientación al cliente, capacidad del empleado y capacitación, capacidad del empleado, capacitación relacionada con el trabajo costo, calidad, flexibilidad, tiempo, gestión humana y su impacto en el desempeño del sistema productivo como un campo fértil para profundizar en la investigación, entre otros.

Con base en esta revisión, se elaboró un formato que fue aplicado a la muestra seleccionada, tal como se especifica en la tabla 1.

\section{Tabla 1.}

Ficha técnica del trabajo de campo.

\begin{tabular}{|l|l|}
\hline Universo & $\begin{array}{l}\text { América Latina: Participación sectorial de las empresas de } \\
\text { menor tamaño homologas a CIIU 5229 datos del Índice de } \\
\text { Desempeño Logístico del Banco Mundial 2017, Colombia, } \\
\text { Paraguay, Nicaragua, Guatemala, Honduras, Haití y Bolivia. }\end{array}$ \\
\hline Muestra & 126 \\
\hline $\begin{array}{l}\text { Ámbito (empresas } \\
\text { de menor tamaño })\end{array}$ & $\begin{array}{l}\text { Empresas de menor tamaño dedicadas a las actividades } \\
\text { logísticas que colaboraron con el estudio. }\end{array}$ \\
\hline Error muestral & $+/-10 \%$ \\
\hline Nivel de confianza & $99 \%, \mathrm{z}=2,57 \mathrm{p}=\mathrm{q}=0,5$ \\
\hline Diseño muestral & $\begin{array}{l}\text { Muestreo aleatorio estratificado con afijación proporcional, } \\
\text { considerando como estrato las empresas de menor tamaño } \\
\text { dedicadas a actividades logísticas en AL. Para la selección } \\
\text { probabilística de la muestra se utilizó el Teorema de } \\
\text { Transformación Integral Inversa. }\end{array}$ \\
\hline $\begin{array}{l}\text { Recolección de } \\
\text { información }\end{array}$ & $\begin{array}{l}\text { A través de la página www.encuestafacil.com o mediante } \\
\text { formato físico. }\end{array}$ \\
\hline Trabajo de campo & Entre julio de 2017 y febrero de 2018 \\
\hline
\end{tabular}

Fuente: elaboración propia.

Para el procesamiento de datos se utilizaron dos métodos estadísticos:

El análisis factorial (afc): es una metodología descriptiva de carácter multivariante que permite la reducción de una gran cantidad de información a un pequeño número de modalidades de variables cualitativas asociadas, con la menor pérdida posible de información. Requiere que los datos representen las respuestas de un grupo de individuos a un conjunto de preguntas. 
- La regresión logística (rl): es una técnica de análisis inferencial utilizada para predecir el resultado de una variable dependiente categórica y dicotómica (solo acepta dos posibles respuestas), es decir, aquella cuyos elementos de variación tienen carácter cualitativo y adopta modalidades de variables explicativas en función de la variable dependiente. Esta técnica valora la contribución de diferentes factores en la ocurrencia de un evento simple (De la Fuente, 2011), cuyos resultados se obtienen comparando el resultado de las modalidades presentes en la tabla, con la casilla de referencia (no presente en la tabla) mediante el criterio de la razón de proporciones.

En el procesamiento de datos se utilizaron los programas estadísticos SPSS (versión 23) y Statgraphics Centurión (versión 16.1.15).

\section{LAS VARIABLES Y EL MODELO UTILIZADO}

Las variables objeto de estudio fueron:

Gestión de operaciones (GO), toma el valor de 1 si la empresa dispone de un operador logístico y 0 en otro caso. Para efectos de este trabajo se utilizó como variable proxy de la gestión de operaciones logística (GO), si la empresa dispone o no de un operador logístico la variable $\boldsymbol{X}_{5}$

Talento Humano (TH) fue dicotomizada tomando el valor de 1 si el talento humano está especializado en asuntos logístico y 0 en otro caso;

Con base en los argumentos planteados en los ítems anteriores se intentó probar la hipótesis:

Ho La probabilidad de ocurrencia de la razón por la cual ocurre un bajo desempeño en la gestión de operaciones logística en América Latina no se puede predecir por la falta de talento humano especializado en logística.

Al respecto,

- Variable independiente: talento humano especializado en asuntos logísticos (TH) se siguen los trabajos de Aguilar, J.A. (2001). Acero, M (2002), Aguezzoul, A. (2014). Arciniegas H. (1998), Ariza,J.E.(1997).Avendaño, G. (2003), Ballou, R. (2004). Bowersox, J.D; Cross J.D; Helferich O.K. (1986), Burbano, E., 
Blanco, L. y Morales, R. (2009), Burtman, J., Bargam, M. (2002), Carrasco, J. (2000), Carrión, A. (1998), Cohen S. y Rousse J. L, (2005), Cooke P. (1997). Correa, A. y Gómez, R. (2009). Chopra, S.l y Meindl, P. (2008), Díaz, García y N. Porcell (2008), Fajardo, H. (2017), García, J.G. (2004), Gallardo, H. (2005), González, J. (2015), Giraldo, C.M., (2000), Harland, C.M., Lamming, R.C., \& Cousins P, D., (1999), Jiménez, E. (2000), Jiménez, J. y Hernández, S. (2002), Kulmala, H.I. (2004). Kirby, C. \& Brosa, N. (2011).

- Variable dependiente: Gestión de Operaciones (GO), se siguen los trabajos de Carman, J.M. (1990), Crosby, P. (1991), Guasch, J. (2011), Handfield, R., \& Nichols, E. L. (2002). Mentzer, J.T., Myers, M.B. y Cheung, M-S. (2004), Millen, R. y Maggard, M. (1997)

Los modelos de elección discreta predicen directamente la probabilidad de la ocurrencia de un suceso que viene definido por los valores de las variables independientes. Como los valores de una probabilidad están entre cero y uno. Las predicciones realizadas con los modelos de elección discreta deben estar acotadas para que caigan en el rango entre cero y uno. El modelo general que cumple esta condición es un caso particular del modelo de regresión múltiple que se denomina modelo de elección discreta, y tiene la forma funcional:

$$
P_{i}=F\left(x_{i}, \beta\right)+u_{i}
$$

Se observa que si $F$ es lineal tenemos el modelo lineal de probabilidad, pero si $F$ es la función de distribución de una variable aleatoria, entonces $P$ varía entre cero y uno de modo seguro.

En el caso particular en que la función $F$ es la función logística estaremos ante el modelo Logit o Regresión Logística, cuya forma funcional será la siguiente:

$$
P_{i}=F\left(x_{i}, \beta\right)+u_{i}=\frac{e^{x_{i} \beta}}{1+e^{x_{i} \beta}}+u_{i}
$$

En el caso particular en que la función $F$ es la función de distribución de una normal unitaria estaremos ante el modelo Probit, cuya forma funcional será la siguiente: 


$$
P_{i}=F\left(x_{i}, \beta\right)+u_{i}=(2 \pi)^{-\frac{1}{2}} \int_{-\infty}^{x_{i} \beta} e^{-\frac{t^{2}}{2}} d t+u_{i}
$$

Sin embargo, si el tipo de modelo de regresión que se analiza opera con variable dependiente binomial (modelo logístico o modelo de regresión logística) será un modelo que permita estudiar si dicha variable discreta depende o no de otra u otras variables. Si una variable binomial de parámetro $p$ es independiente de otra variable $X$, se cumple $(\mathrm{p} \mid X=x)=p$, para cualquier valor de $x$ de la variable $X$. Por consiguiente, un modelo de regresión con variable dependiente binomial y una única variable independiente $X$ se materializa en una función en la que $p$ $p$ aparece dependiendo de $X$ y de unos coeficientes cuya investigación permite abordar la relación de dependencia.

Para una única variable independiente $X$ el modelo de regresión logística toma la forma:

$$
\ln (p / q \mid X=x)=\alpha_{0}+\alpha_{1} X
$$

O de forma simplificada:

$$
\ln (p / q)=\alpha_{0}+\alpha_{1} X
$$

Donde $\ln$ significa logaritmo neperiano, $\alpha_{0}$ y $\alpha_{1}$ son constantes y $X$ una variable que puede ser aleatoria o no, continua o discreta. Este modelo se puede fácilmente generalizada para $\mathrm{k}$ variables independientes, dando lugar al modelo logístico múltiple, que expresa como sigue:

$$
\ln (p / q)=\alpha_{0}+\alpha_{1} X_{1}+\alpha_{2} X_{2}+\cdots \alpha_{k} X_{k}
$$

Hemos definido el modelo logístico como el logaritmo de odds para el suceso que representa la variable aleatoria binomial puntual dependiente del modelo.

$$
\text { Odds }=\text { Probabilidad de éxito } / \text { Probabilidada de fracaso }=p / q
$$

Hay varias razones para plantear el modelo con el logaritmo de odds, en lugar de plantearlo simplemente con la probabilidad de éxito o con el odds. En primer lugar, el campo de variación de $\ln (p l q)$ es todo el campo real (de $-\infty$ a 
$\infty$ ), mientras que para $p$ el campo es sólo de 0 a 1 y para $p / q$ es de 0 a $\infty$. Por tanto, con el modelo definido en función del logaritmo de odds no hay que poner restricciones a los coeficientes que complicarían su estimación. Por otro lado, y más importante, en los modelos en función del logaritmo de odds los coeficientes son fácilmente interpretables en términos de independencia o asociación entre las variables, como se verá más adelante.

El modelo logístico se puede escribir de otras formas equivalentes que para ciertas aplicaciones son más cómodas de manejar. Tenemos:

$$
\begin{gathered}
\ln \left(\frac{p}{q}\right)=\alpha_{0}+\alpha_{1} X \Leftrightarrow \ln \left(\frac{p}{1-p}\right)=\ln \left(\frac{p}{q}\right)=\alpha_{0}+\alpha_{1} X \Leftrightarrow \frac{p}{1-p}=e^{\alpha_{0}+\alpha_{1} X} \Leftrightarrow \\
p=\frac{e^{\alpha_{0}+\alpha_{1} X}}{1+e^{\alpha_{0}+\alpha_{1} X}} \Leftrightarrow p=\frac{1}{1+e^{-\left(\alpha_{0}+\alpha_{1} X\right)}}
\end{gathered}
$$

Estas dos últimas expresiones, si son conocidos los coeficientes $\alpha_{0}$ y $\alpha_{l}$, permiten calcular directamente la probabilidad del proceso binomial para los distintos valores de la variable $X$.

A la función $f(z)=\frac{1}{1+e^{-z}}$ se le denomina función logística. El modelo de regresión logística modeliza la probabilidad de un proceso binomial como la función logística de una combinación lineal de la variable dependiente.

El modelo de regresión logística múltiple tendrá la expresión:

$$
p=\frac{1}{1+e^{-\left(\alpha_{0}+\alpha_{1} X_{1}+\alpha_{2} X_{2}+\cdots+\alpha_{k} X_{k}\right)}}
$$

Finalmente, para abordar el tema de la estimación de los coeficientes, el método de los mínimos cuadrados, clásico en la estimación de los coeficientes de los modelos de regresión, no es aplicable al modelo logístico, dado que, dicho método se basa en la normalidad de la variable dependiente, que en este caso no se cumple. Por otra parte, cuando $q=0$, es imposible calcular $\ln (p / q)$. Se tratará entonces de utilizar el método de máxima verosimilitud.Se consideró ando el caso más simple con una sola variable independiente $X$. tomamos una muestra 
de $n$ observaciones $\left(y_{i}, x_{i}\right)$ para la variable puntual binomial dependiente $Y$ y para la variable independiente $X$. La variable $Y$ toma valores $\mathrm{y}_{i}$ que sólo pueden se 1 con probabilidad $p_{i}$ o 0 con probabilidad 1- $p_{i}$. Como $x_{i}$ depende de $p_{i}$ a través del modelo logístico tenemos:

$$
p=\frac{e^{\alpha_{0}+\alpha_{1} X_{i}}}{1+e^{\alpha_{0}+\alpha_{1} X_{i}}}
$$

La función de verosimilitud para una variable binomial puntual es:

$$
L\left(p_{i} \mid y_{i}\right)=\left(p_{i}\right)^{y_{i}}(1-p)^{1-y_{i}}
$$

Y para $n$ observaciones independientes la función de verosimilitud de la muestra será:

$$
L\left(p_{1}, \ldots, p_{n} \mid y_{1}, \ldots, y_{n}\right)=\prod_{i=1}^{n}\left(p_{i}\right)^{y_{i}}(1-p)^{1-y_{i}}
$$

y al representar $p_{i}$ por el modelo logístico tendremos ya la expresión de la función de verosimilitud para la muestra como función de los parámetros a estimar:

$$
\begin{gathered}
L\left(p_{1}, \ldots, p_{n} \mid y_{1}, \ldots, y_{n}\right)=\prod_{i=1}^{n}\left(\frac{e^{\alpha_{0}+\alpha_{1} X_{i}}}{1+e^{\alpha_{0}+\alpha_{1} X_{i}}}\right)^{y}\left(1-\frac{e^{\alpha_{0}+\alpha_{1} X_{i}}}{1+e^{\alpha_{0}+\alpha_{1} X_{i}}}\right)^{1-y_{i}}= \\
=\prod_{i=1}^{n}\left(\frac{e^{\alpha_{0}+\alpha_{1} X_{i}}}{1+e^{\alpha_{0}+\alpha_{1} X_{i}}}\right)^{y}\left(\frac{e^{\alpha_{0}+\alpha_{1} X_{i}}}{1+e^{\alpha_{0}+\alpha_{1} X_{i}}}\right)^{1-y_{i}}=\frac{e^{\alpha_{0} \sum_{i=1}^{n} y_{i}+\alpha_{1} \sum_{i=1}^{n} x_{i y_{i}}}}{\prod_{i=1}^{n} 1+e^{\alpha_{0}+\alpha_{1} X_{i}}} \\
=L\left(\alpha_{0}, \alpha_{1}\right)
\end{gathered}
$$

y como suele ser usual en máxima verosimilitud, maximizaremos el logaritmo de la función $L\left(\alpha_{0}, \alpha_{l}\right)$ en vez de la función misma. Los parámetros estimados del modelo serán los valores de $\alpha_{0}$ y $\alpha_{1}$ que maximicen la función $\operatorname{Ln} L\left(\alpha_{0}, \alpha_{1}\right)$.

En este sentido, para la estimación por intervalos y contrastes de hipótesis sobre los coeficientes, partimos del teorema central del límite, los estimadores 
por máxima verosimilitud de los parámetros del modelo logístico son asintóticamente normales y su matriz de varianzas covarianzas es calculable a partir del algoritmo de maximización de la función de verosimilitud (Método de Newton Rhampson).

De esta forma, un intervalo de confianza al $(1-\alpha) \%$ para el estimador del coeficiente $\alpha_{i}$ del modelo será:

$$
\hat{\alpha}_{i} \pm Z_{\alpha / 2} \hat{\sigma}\left(\hat{\alpha}_{i}\right)
$$

Hay que tener presente que los estimadores habituales que miden asociación entre variables son los odds ratio, por tanto, interesa dar los intervalos de confianza para los odds ratio, que evidentemente serán:

$$
e^{\widehat{\alpha}_{i} \pm Z_{\alpha / 2} \widehat{\sigma}\left(\widehat{\alpha}_{i}\right)}
$$

El estadístico para el contraste será:

$$
\begin{gathered}
H_{0}: \alpha_{i}=a \\
H_{1}: \alpha_{i} \neq a \\
Z=\frac{\hat{\alpha}_{i}-a}{\hat{\sigma}\left(\hat{\alpha}_{i}\right)} \rightarrow N\left(a, \hat{\sigma}\left(\hat{\alpha}_{i}\right)\right)
\end{gathered}
$$

y con región crítica $|Z|>Z_{\alpha / 2}$

También suele utilizarse para el contraste el estadístico de Wald definido como $W=Z^{2}$ y cuya distribución es una chi-cuadrado con 1 grado de libertad. La región crítica de este contraste es $W=\chi^{2} \alpha$.

En el modelo logístico es muy interesante contrastar la hipótesis $\alpha_{i}=0$ para $i=1, \ldots, k$, porque, no rechazar esta hipótesis para un valor de $i$, implica que la variable $Y$ no depende $X_{i}$, y por lo tanto esta última no debería figurar en el modelo.

También suele utilizarse el contraste de la razón de verosimilitudes, basado en el estadístico $-2 \log \left(L_{\delta} L_{\nu}\right)$ donde $L_{0}$ es el máximo de la función de la verosimilitud bajo la hipótesis nula y $L_{1}$ es el máximo de la función de verosimilitud bajo 
la hipótesis alternativa. Este estadístico tiene una distribución chi-cuadrado con grados de libertad igual al número de parámetros bajo la hipótesis nula. Si se elige la función $F$ como la función de distribución de una Normal $(0,1)$, el modelo lineal general:

$$
P_{i}=F\left(x_{i} \beta\right)+u_{i}
$$

se denomina modelo Probit, cuyos parámetros $\boldsymbol{\beta}$ admiten estimación por máxima verosimilitud dado por:

$$
F\left(x_{i}, \beta\right)=(2 \pi)^{-\frac{1}{2}} \int_{-\infty}^{x_{i} \beta} e^{-\frac{t^{2}}{2}} d t
$$

En el caso de que

$$
F\left(x_{i}, \beta\right)=\frac{e^{x_{i} \beta}}{1+e^{x_{i} \beta}}
$$

Estaríamos ante el modelo Logit dado que el logaritmo de la razón de probabilidades es lineal, tanto en las variables como en los parámetros. La estimación de estos puede realizarse mediante el método de máxima verosimilitud (Green 2001) quedando el modelo logita así:

\section{RESULTADOS}

Los resultados se pueden resumir en siete (7) puntos así:

1. Se probó la hipótesis que predice que la probabilidad de ocurrencia de la razón por la cual ocurre un bajo desempeño en la gestión de operaciones logística en América Latina no se puede predecir por la falta de talento humano especializado en logística.

2. Los test la prueba de ómnibus, el resumen del modelo y el modelo de elección discreta en cada una de las tablas de referencia 1,2,3 y finalmente la Prueba de Hosmer y Lemeshow para validar el modelo. Tabla 1 


\section{Tabla 1.}

Pruebas ómnibus de coeficientes de modelo

\begin{tabular}{|rl|r|r|c|}
\hline & & Chi-cuadrado & gl & \multicolumn{1}{c|}{ Sig. } \\
\hline Paso 1 & Paso & 24,684 & 2 & 0,000 \\
& Bloque & 24,684 & 2 & 0,000 \\
& Modelo & 24,684 & 2 & 0,000 \\
\hline
\end{tabular}

Fuente autor salida spss v23

3. El modelo de regresión logística múltiple tuvo la forma:

$$
p=\frac{1}{1+e^{-\left(\alpha_{0}+\alpha_{1} X_{1}+\alpha_{2} X_{2}+\cdots+\alpha_{k} X_{k}\right)}}
$$

Obteniéndose el modelo $X^{\prime} \beta=1,511-2,185 X_{5}$

El modelo se validó con los siguientes procesos tabla 2 y 3

\section{Tabla 2.}

\section{Resumen del modelo}

\begin{tabular}{|l|r|r|r|}
\hline Paso & $\begin{array}{c}\text { Logaritmo de la } \\
\text { verosimilitud }-2\end{array}$ & $\begin{array}{c}\text { R cuadrado de } \\
\text { Cox y Snell }\end{array}$ & $\begin{array}{c}\text { R cuadrado de } \\
\text { Nagelkerke }\end{array}$ \\
\hline 1 & $40,657^{\text {a }}$ & 0,390 & 0,534 \\
\hline
\end{tabular}

a. La estimación ha terminado en el número de iteración 5 porque las estimaciones de parámetro han cambiado en menos de 0,001 .

b. Fuente autor salida spss v23.

\section{Tabla 3.}

\section{Resultados del modelo}

\begin{tabular}{|c|c|r|r|r|r|r|}
\hline Variables & B & \multicolumn{1}{c|}{$\begin{array}{c}\text { Error } \\
\text { estándar }\end{array}$} & Wald & gl & Sig. & Exp(B) \\
\hline$X_{5}$ & $-2,185$ & 0,816 & 7,164 & 1 & 0,007 & $0,112 * * *$ \\
\hline intercepto & 1,511 & 0,631 & 5,741 & 1 & 0,017 & $4,531 * * *$ \\
\hline
\end{tabular}

Fuente autor salidas spss v23 con $\mathrm{P}$ value $<0.05$ sig.

4. Para analizar cuáles son las variables que mayor influencia tienen en el TH se realizó un análisis de RL, en el que la variable respuesta es GO, que se 
transformó en una variable dicotómica en la que solo se tomaron dos opciones: uno (1), sí TH es especializado, y cero (0), en caso contrario no tiene intención de crear empresa.

5. Se pudo observar que el valor del coeficiente -2.185 significa que, dejando todas las demás variables constantes, la falta de talento humano especializado en logística disminuiría la probabilidad del desempeño de la gestión de operaciones logística en 2.18 veces en las empresas de AL.

6. Para validar el modelo utilizamos el test de Hosmer y Lemesshow (2000), con Chi-cuadrado $0,041 \mathrm{y}$ un valor $\mathrm{p}$-valor de 0,041 , confirma que el valor $\mathrm{R}$ cuadrado de Nagelkerke explica el 53,4\% de la varianza del desempeño de la gestión de operaciones logística tabla 4.

\section{Tabla 4.}

Prueba de Hosmer y Lemeshow

\begin{tabular}{|l|r|r|c|}
\hline Paso & Chi-cuadrado & gl & Sig. \\
\hline 1 & 6,405 & & 0,041 \\
\hline
\end{tabular}

Fuente autor salidas spss v23

7. El valor EXP (B) de 0,112 con punto de corte 1, para este caso, la puntuación de EXP (B) es menor a uno, esto indica que, la falta de talento humano especializado en asuntos logísticos, disminuye el desempeño en la gestión de operaciones logística. Esto se confirma además por la puntuación de Wald que para este modelo fue de 7,164 gl $1 \mathrm{y}$ un p-valor de 0,007 menor al 0. 001.Con esto comprobamos la hipótesis de que la falta talento humano especializado permite predecir en un $86 \%$ la probabilidad de concurrencia de la razón por la cual hay un bajo desempeño en la gestión de operaciones logística en la pequeña empresa en AL.

\section{CONCLUSIONES.}

El talento humano es un factor clave en el desempeño de la gestión de operaciones (GO) para la trazabilidad de los procesos, en la logística de abastecimiento, en producción, programación de producción y logística distribución, para la muestra analizada. En segundo lugar, los resultados sugieren 
que $\mathrm{TH}$ genera un efecto significativo en la GO para $\mathrm{AL}$ lo que posiblemente indicaría que las firmas de menor tamaño deberían poner mayor énfasis en mantener capacitados a los empleados en procesos y asuntos logísticos.

Para el caso colombiano, en materia logística en el ranking de la región, al pasar de la posición 10 a la 13 entre los años 2016 y 2017, del Índice de Desempeño Logístico del Banco Mundial 2017, y en relación con Latinoamérica, Colombia solo supera a Paraguay, Nicaragua, Guatemala, Honduras, Haití y Bolivia.

$\mathrm{Al}$ respecto, de esto, los parámetros internacionales, por ejemplo, del Banco Mundial, solo consideran el bajo desempeño logístico, explicado por cuatro factores: la eficiencia de las aduanas, baja capacidad de los envíos de llegar a su destino a tiempo, la falta de seguimiento y localización de las mercancías y la calidad de los servicios logísticos, pero, ¿del talento humano y sus efectos en el desempeño logística nada o casi nada se dice? Al respecto: Jayaram y Xu, (2016) evalúan los determinantes de la calidad y el desempeño de la eficiencia en las operaciones de servicio, estos autores proponen un modelo hipotético, utilizando una muestra de 249 empresas de servicios chinos, aplicando como metodología las ecuaciones estructurales y análisis de regresión jerárquica. Los resultados encontrados por ello indican que los factores de gestión del conocimiento de orientación al cliente, capacidad del empleado y capacitación relacionada con el trabajo tuvieron una influencia variable en las dimensiones de calidad y eficiencia del rendimiento del sistema de servicio. Además, las facetas de conocimiento interno de la capacidad del empleado y la capacitación relacionada con el trabajo tuvieron efectos complementarios al inducir un mejor desempeño.

Jayaram y Vickery (1999). En un estudio relacional respecto al "The impact of human resource management practices on manufacturing performance. Journal of Operations Management"estudian la relación entre diversas prácticas de gestión de recursos humanos (compromiso de la alta dirección, comunicación de objetivos, entrenamiento formal, equipos de trabajo multifuncionales, trabajos amplios, entrenamiento cruzado, autonomía, impacto del empleado, administración de relaciones laborales y organizaciones abiertas) y el desempeño en manufactura a través de cuatro prioridades competitivas (costo, calidad, flexibilidad y tiempo) los autores concluyen relaciones significativas entre estas variables. 
Da Silveira \& Sousa (2010), evalúan el efecto sobre cuatro prioridades (costo, calidad, entregas, flexibilidad) y comprobó que estas prácticas tuvieron efecto significativo sobre el costo, pero no sobre las demás, a pesar que la literatura sugiere ampliamente un efecto positivo, por lo que estos autores sugirieron las posibles razones por la cual no encontraron relaciones. Al respecto, Furlan. \& Dal (2011) encuentran relaciones significativas entre la gestión humana y su impacto en el desempeño del sistema productivo como un campo fértil para profundizar en la investigación.

Goodridge (1986) plantean que la administración de operaciones británica debería poner mayor énfasis en mantener las relaciones con los empleados, además consideran que la tecnología, impactan significativamente al empleo y estas interacciones generan efectos sobre la gestión de operaciones e indican que es necesario pasar de una orientación de control a una de compromiso en el lugar de trabajo.

Patrick y Dul, (2010) trabajaron en "Human factors: spanning the gap between OM and HRM", probaron la hipótesis, respecto a, la aplicación del conocimiento de los factores humanos (FC) puede mejorar el rendimiento del sistema de operaciones y el bienestar humano. Los autores realizan una revisión sistemática utilizando una base de datos general y dos especializadas para identificar estudios empíricos que abordan tanto los efectos humanos como los del sistema operativo al examinar los aspectos de diseño del sistema operativo de fabricación. El trabajo mostró una convergencia entre efectos humanos y efectos del sistema incluyeron calidad, productividad, rendimiento de implementación de nuevas tecnologías y también más efectos "intangibles" en términos de comunicación y cooperación mejoradas. Los efectos humanos incluyeron la salud de los empleados, las actitudes, la carga de trabajo física y la "calidad de la vida laboral"

En suma, los resultados de este trabajo deberían tomarse con prevención, dado algunos factores limitantes, como son, el uso de modelos estadísticos para aproximarnos en comprender realidades o fenómenos de las Ciencias Sociales. 


\section{REFERENCIAS}

(1) Acero, M (2002): La verdadera dimensión de las cadenas de suministros. En: Zona logística No.22. Pg 22-24.

(2) Aguilar, J.A. (2001). Subcontratación de servicios logísticos. Barcelona.

(3) Aguezzoul, A. (2014). Third-party logistics selection problem: A literature review on criteria and methods. Omega, 49 (3) 69-78.

(4) Ahmad, S. \& Schroeder, R.G. (2003). The impact of human resource management practices on operational performance: recognizing country and industry differences. Journal of Operations Management

(5) Arciniegas H. (1998): Estudio de la caracterización logística en Colombia. Mesa sectorial.

(6) Avendaño, G. (2003) Costos en la cadena de Distribución física Internacional DFI. En: Zona Logística. Vol 16. Pg 42-45

(7) Ballou, R. (2004). Business Logistics Management. Prentice Hall, USA.

(8) Ballou, R. (1999): Business Logistic Management. Planing, organizing and controlling the supply Chain. 4 ed. Prentice Hall, New Jersey.

(9) Beamon (1998). Supply chain design and analysis: models and methods. International Journal of Production Economic, 55, pp. 281-294.

(10) Bowersox, J.D; Cross J.D; Helferich O.K. (1986): Logistical Management. A system integration of physical distribution, manufacturing support and materials procurement.

(11) Burbano, E., Blanco, L. y Morales, R. (2009). La logística, una prolongación del servicio al cliente. Revista Gestión y Desarrollo. Volumen 6. No. 2. Julio-diciembre de 2009. Universidad de San Buenaventura. Cali.

(12) Burtman, J., Bargam, M. 2002. A pain in the chain: en Harvard Business Review. Mayo Pg 31-44.

(13) Carman, J.M. (1990). "Consumer perceptions of service quality: an assessment of the SERVQUAL dimensions”. Journal of Retailing, Vol. 66 (Primavera), pp. 33-55

(14) Carrasco, J. (2000): Evolución de los enfoques y conceptos de la logística "Su impacto en la dirección y la gestión de las organizaciones”. Economía Industrial. No. 331. Pp.17-34.

(15) Carrión, A. (1998): Nuevo modelo de organización en el área logística del hospital de Albacetel. La gestión de suministros más próxima a la actividad asistencial. 
(16) Cohen S. y Rousse J. L, (2005): Avantage Supply Chain, Editions d'organisation, Paris.

(17) Correa, A. y Gómez, R. (2009). Tecnologías de la información en la cadena de suministro. Dyna, Año 76, Nro. 157, pp. 37-48. Medellín, marzo de 2009. ISSN 0012-7353

(18) Crosby, P. (1991). La calidad no cuesta. El arte de cerciorarse de la calidad. CECSA, Méjico.

(19) Chopra, S.1 y Meindl, P. (2008). Administración de la cadena de suministro: Estrategia, planeación y operación. No. 3. México.

(20) Da Silveira, G.J.C. \& Sousa, R.S. (2010). Paradigms of choice in manufacturing strategy Exploring performance relationships of fit, best practices, and capability-based approaches. International Journal of Operations \& Production Management.

(21) Debreu, G. (1951):” The Coefficient of Resource Utilization”.Econometrica,19;14-22.

(22) De la Fuente, S. (2011). Regresión Logística. Madrid: Universidad Autónoma de Madrid.

(23) Díaz, García y N. Porcell (2008). Las Pymes: costos en la cadena de abastecimiento. Revista Escuela de Administración de Negocios, mayo-agosto 63: 5-22.

(24) Fajardo, H. (2017). Análisis del sector de operadores logísticos en Colombia, para la creación de un modelo de selección de servicios logísticos utilizando la metodología AHP. Tesis de Investigación presentada como requisito parcial para optar al título de: Magister en Ingeniería Industrial. Universidad Nacional. Bogotá.

(25) Farrel, M.J. (1957): “The Measurement of Productive Efficiency". Journal of the Royal Statistical Society, Series A, 120, Part III, 253-290.

(26) Furlan, A., Vinelli, A. \& Dal Pont, G. (2011). Complementarity and lean manufacturing bundles: an empirical analysis. International Journal of Operations \& Production Management.

(27) García, J.G. (2004): Logística una herramienta en los negocios. En Mc Graw Hill. New York.

(28) Gallardo, H. (2005). Operadores Logísticos, Tendencias y Perspectivas. Chile.

(29) Giraldo, C.M., (2000): Logística, instrumento de productividad y competitividad. Revista ANDI Nov-Dic. Pg 92-103.

(30) González, J. (2015). Contratación logística en Colombia: implementación de un operador logístico integral. Semestre Económico, volumen 18, No. 38, pp. 215-238 • ISSN 01206346, julio-diciembre de 2015, Medellín, Colombia.

(31) Goodridge, Mark (1986) "Operations Management of Human Resources in the 1990s", International Journal of Operations \& Production Management, Vol. 6 Issue: 4, pp.42-60. 
(32) Guasch, J. (2011). La logística como motor de la competitividad en América Latina y el Caribe. Banco Interamericano de desarrollo. Recuperado el 08 de Julio de 2017, en http:// publications.iadb.org/handle/11319/5091

(33) Handfield, R., \& Nichols, E. L. (2002). Supply Chain Redesign: Transforming Supply Chains Into Integrated Value Systems. The United States: FT Press.

(34) Harland, C.M., Lamming, R.C., \& Cousins P, D., (1999): Developing the concept of supply strategy. International Journal of Operations \& Production Management. Vol 19. No. 7.pg. 650-673.

(35) Hosmer, D. W. \& Lemeshow, S. (2000), Applied Logistic Regression, Jonh Wiley.

(36) Jiménez, E. (2000). Propuesta Doctoral “Análisis de las cadenas de suministro en el marco de la competitividad internacional”. UNAM, 2000 (documento inédito).

(37) Jayanth Jayaram, Kefeng Xu, (2016) "Determinants of quality and efficiency performance in service operations", International Journal of Operations \& Production Management, Vol. 36 Issue: 3 , pp.265-285.

(38) Jiménez, J. y Hernández, S. (2002). Marco conceptual de la cadena de suministro: Un nuevo enfoque logístico. Secretaría de Comunicaciones y Transporte. Instituto Mexicano de Transporte. Publicación Técnica No. 215. Safandila.

(39) Kirby, C. \& Brosa, N. (2011). La logística como factor de competitividad de las Pymes en las Américas. Banco Interamericano de Desarrollo. Santo Domingo, República Dominicana.

(40) Lambert, Douglas; Stock, James R; Ellram, Lisa M. (1998). Fundamentals of logistics management. McGraw Hill. New York

(41) Langley, C.J. (1986). Evolution of logistics concepts. En: Journal of Bussines Logistics No 7 (2) pg 1-13.

(42) Patrick Neumann, W. Dul, J (2010) "Human factors: spanning the gap between OM and HRM", International Journal of Operations \& Production Management, Vol. 30 Issue: 9, pp.923-950.

(43) Servera, D., Gil, I. y Fuentes, M. (2009). La influencia de la calidad de servicio logístico en la lealtad. Un análisis del papel moderador de las TIC. Investigaciones Europeas de Dirección y Economía de la Empresa Vol. 15, No 3, 2009, pp. 33-54, ISSN: 1135-2523

(44) Sarache,W \& Hernandez, G.(S/A): Estrategia de Operaciones y enfoque logístico: Punto de partida en el mejoramiento de Sistema de Producción. 